\title{
Predictive value of soluble urokinase plasminogen activator receptor, soluble ST2, and IL-33 in bronchopulmonary dysplasia
}

\author{
Turan Tunc', Ferhat Cekmez', Sukran Yildirim², Ozgul Bulut² , Zeynep Ince², Mehmet Saldir ${ }^{3}$, Gokhan Aydemir ${ }^{4}$, Halil Yaman $^{5}$ and \\ Asuman Coban²
}

BACKGROUND: Bronchopulmonary dysplasia (BPD) remains an important complication of preterm births. The soluble form of ST2 (sST2), interleukin-33 (IL-33), and soluble form of the urokinase plasminogen activator receptor (suPAR) have attracted increasing attention as biomarkers for different diseases. The aim of the current study was to assess the predictive value of plasma sST2, IL-33, and suPAR levels in patients with risk of BPD development.

METHODS: A total of 38 babies were studied prospectively on delivery to the neonatal intensive care unit. Serum levels of IL-33, sST2, and suPAR were measured using enzyme-linked immunosorbent assay. Serum samples were collected from umbilical cord (at the time of delivery, termed CB) and peripheral blood (on day 14, termed PB).

RESULTS: Levels of suPAR (PB-suPAR) and SST2 (PB-sST2) in the peripheral blood of the BPD group were significantly higher than the corresponding levels in the non-BPD group $(P<0.001$, $P=0.028$, respectively. There was a statistically significant correlation between PB-suPAR levels and the severity of BPD $(P<0.001))$ when the suPAR results were analyzed using the receiver operating characteristic curve.

CONCLUSION: PB-sUPAR and PB-SST2 levels are sensitive and specific independent predictive biomarkers in preterm babies with BPD.

B ronchopulmonary dysplasia (BPD) is a chronic lung disease associated with preterm babies who require mechanical ventilation and oxygen therapy for acute respiratory distress (1). BPD remains a serious and common problem in very-low-birth-weight infants despite the use of antenatal steroids and postnatal surfactant therapy to decrease the incidence and severity of respiratory distress syndrome (RDS) $((2-4)$. The incidence of BPD is directly proportional to the degree of prematurity. BPD has a complex and multifactorial etiology, including oxygen toxicity, preterm delivery, hypoxia/ hyperoxia, infection, and inflammation (5-7). Many studies have suggested that BPD is a result of imbalance between proinflammatory and anti-inflammatory mechanisms $(8,9)$.

The ST2 gene, which produces a soluble secreted form (sST2) and a transmembrane form (ST2L) of the IL-1 receptor, is expressed in several cells, including Th2 cells, mast cells, and macrophages (10). Interleukin-33 (IL-33) was identified as a new member of the IL-1 cytokine family. It has been described as a modulator of inflammation, mediating Th2 immune responses. It has also been detected that IL-33 is involved in the pathogenesis of chronic inflammatory diseases (11). Recently, it has been shown that IL-33 can bind to ST2L and can thereby trigger Th2-associated responses. Soluble ST2, which is mainly secreted by fibroblasts, has been suggested to act as a decoy receptor by binding IL-33, thereby inhibiting signaling by ST2L (12). Elevated sST2 levels have been reported in sepsis, asthma, and acute myocardial infarction. A significant positive correlation between sST2 levels and severity of these medical conditions has been shown (13).

The urokinase plasminogen activator (uPA) and the receptor of uPA (uPAR) exist in both endothelial and immune cells (i.e., monocytes and activated T cells), and uPA increases the conversion of plasminogen to plasmin. However, it also has various effects on the regulation of cell migration, angiogenesis, proliferation, and adhesion (14). A soluble form of UPAR ( into body fluids, including plasma and urine (15). A strong association has been shown between high suPAR levels and poor prognosis of several cancer types and infectious diseases (16). Further, a recent study has suggested that suPAR is positively associated with the risk of developing diabetes and cardiovascular disease and may be a general marker of low-grade inflammation responsible in the pathogenesis of both diseases (17). Another study has suggested that suPAR is associated with subclinical organ damage independently from traditional risk factors (18).

Because early prediction of BPD development is paramount and can facilitate targeting of prophylactic interventions, in 
this study, we aimed to explore whether plasma sST2, IL-33, and suPAR levels can have a predictive value for BPD.

\section{RESULTS}

A total of 44 preterm infants were included in the study, but six of them were dead due to several reasons. Four of six babies died within the first $2 \mathrm{wk}$ of life due to early-onset sepsis (2) and respiratory failure (2), and the remaining two babies died of necrotizing enterocolitis at the end of the third week. These babies were excluded from the study because they were dead before day 28 , and so it was not clear whether they would have $\mathrm{BPD}$ or not. Of the 38 patients included in the study, 13 were in the non-BPD group and 25 were in the BPD group. Of the latter 25 patients, 7 were in the mild, 10 were in the moderate, and 8 were in the severe BPD groups.

The demographic findings and the plasma levels of sST2, IL-33, and suPAR in the BPD and non-BPD groups are summarized in Table 1. There were no significant differences between the BPD and the non-BPD groups in terms of demographic findings consisting of gestational age, birth weight, gender,

Table 1. The demographic findings and levels of sST2, IL-33, and suPAR in BPD and non-BPD groups

\begin{tabular}{|c|c|c|c|}
\hline & $\begin{array}{l}\text { Non-BPD } \\
\text { group }\end{array}$ & $\begin{array}{l}\text { BPD } \\
\text { group }\end{array}$ & $P$ \\
\hline & $n=13(\%)$ & $n=25(\%)$ & value \\
\hline Birth weight $(\mathrm{g})$, mean $\pm \mathrm{SD}$ & $986 \pm 148$ & $956 \pm 154$ & 0.07 \\
\hline Gestational age (wk), mean \pm SD & $28.6 \pm 1.6$ & $28.2 \pm 1.9$ & 0.08 \\
\hline Gender (boys) & $5(38)$ & $17(68)$ & 0.10 \\
\hline $\begin{array}{l}\text { Antenatal betamethasone } \\
\text { usage }\end{array}$ & $11(85)$ & $19(76)$ & 0.69 \\
\hline PPROM & $2(15)$ & $15(60)$ & $0.02^{*}$ \\
\hline Chorioamnionitis & - & 6 & 0.07 \\
\hline Early sepsis & $1(7)$ & $2(8)$ & 1.00 \\
\hline Late sepsis & $2(15)$ & $9(36)$ & 0.27 \\
\hline Maternal antibiotic usage & $2(15)$ & $5(20)$ & 0.14 \\
\hline RDS & $8(61)$ & $25(100)$ & $0.003^{*}$ \\
\hline $\begin{array}{l}\text { Duration of mechanical } \\
\text { ventilation (d) }\end{array}$ & $2 \pm 2$ & $11 \pm 4$ & $0.01^{*}$ \\
\hline PDA & $3(23)$ & $16(64)$ & $0.04^{*}$ \\
\hline NEC & $1(7)$ & $7(28)$ & 0.22 \\
\hline CB-IL33: median (IQR) (ng/ml) & $0.98(0.57-3.06)$ & $1.26(0.50-4.48)$ & 0.26 \\
\hline PB-IL33: median (IQR) (ng/ml) & $0.60(0.49-2.61)$ & $1.30(0.47-9.66)$ & 0.08 \\
\hline CB-sST2: median (IQR) (ng/ml) & $0.39(0.13-1.26)$ & $0.60(0.22-3.51)$ & 0.17 \\
\hline PB-sST2: median (IQR) (ng/ml) & $0.32(0.21-1.25)$ & $0.75(0.12-3.12)$ & $0.03^{*}$ \\
\hline CB-suPAR: median (IQR) (ng/ml) & $1.16(0.43-2.93)$ & $1.97(0.51-4.9)$ & 0.16 \\
\hline PB-suPAR: median (IQR) (ng/ml) & $0.79(0.38-2.27$ & $2.33(1.06-5.43)$ & $0.001^{*}$ \\
\hline
\end{tabular}

$\mathrm{BPD}$, bronchopulmonary dysplasia; $\mathrm{CB}$, cord blood; $\mathrm{Cl}$, confidence interval; IL, interleukin; IQR, interquartile range; NEC, necrotizing enterocolitis; PAR, plasminogen activator receptor; PB, peripheral blood; PDA, patent ductus arteriosus; PPROM, preterm premature rupture of membranes; RDS, respiratory distress syndrome; sST2, soluble form of sST2; sUPAR, soluble form of the urokinase plasminogen activator receptor.

${ }^{*} P<0.05$. antenatal betamethasone usage, chorioamnionitis, early or late sepsis, maternal antibiotic usage, and necrotizing enterocolitis. However, in the BPD group, the frequencies of preterm premature rupture of membranes (PPROM), patent ductus arteriosus, duration of mechanical ventilation, and RDS were significantly higher than the frequencies in the non-BPD group. There were no significant differences between the BPD and the non-BPD groups in the CB-IL33, PB-IL33, CB-sST2, and CB-suPAR levels. However, PB-sST2 and PB-suPAR levels in the BPD group were significantly higher than the levels in the non-BPD group (median $=0.32 \mathrm{ng} / \mathrm{ml}$, interquartile range $(\mathrm{IQR})=0.21-1.25 \mathrm{ng} / \mathrm{ml}$ vs. median $=0.75 \mathrm{ng} / \mathrm{ml}, \mathrm{IQR}=$ $0.12-3.12 \mathrm{ng} / \mathrm{ml}, P=0.028 ;$ median $=0.79 \mathrm{ng} / \mathrm{ml}, \mathrm{IQR}=0.38$ $2.27 \mathrm{ng} / \mathrm{ml}$ vs. median $=2.33 \mathrm{ng} / \mathrm{ml}, \mathrm{IQR}=1.06-5.43 \mathrm{ng} / \mathrm{ml}$; $P<0.001$, respectively).

There was a positive, moderate, and statistically significant correlation between PB-suPAR levels and the severity of BPD (rho $=0.659, P<0.001$ ) (Figure 1 ). The optimum diagnostic cutoff point was $1.55 \mathrm{ng} / \mathrm{ml}$, the area under the curve was $0.902, P<0.001$ (95\% confidence interval $(\mathrm{CI})=$ $0.80-0.99)$, sensitivity was $88 \%(95 \% \mathrm{CI}=86.8-89.2)$, specificity: $84.6 \%$ (95\% CI $=83.4-85.8$ ), positive predictive value: $78 \%$ (95\% CI $=77.4-79.8)$, and negative predictive value: 91.7\% (95\% CI $=90.5-92.9)$ (Figure 2a). We found that an increase of $1 \mathrm{ng} / \mathrm{ml}$ in PB-suPAR levels increased the risk of BPD 12.92-fold (95\% CI $=2.52-66.18, P=0.002$ ). The risk of BPD development in elevated PB-suPAR level patients $(>1.55 \mathrm{ng} / \mathrm{ml})$ was 40.33 -fold higher than the risk in those with lower levels $(<1.55 \mathrm{ng} / \mathrm{ml})(95 \% \mathrm{CI}=5.85-277.88$, $P<0.001)$.

There was a statistically significant difference between the subgroups (mild, moderate, and severe) of BPD according to the suPAR levels. To determine the group or groups that cause the main difference in the statistical analysis, posthoc analyses were performed. Thus, we found that the difference originates from the severe BPD group and that the suPAR levels were higher in this group than in the mild and moderate BPD groups ( $P=0.003$, and $P=0.009$, respectively). Due to the small sample size in our study, patients with mild BPD who do not require any therapeutic interventions and those without BPD were considered as a single group $(n=20)$; on the other hand, patients with moderate and severe BPD were both considered as the second group $(n=18)$. When receiver operating characteristic analysis was performed for these two groups, the area under the curve was found to be 0.861 , with $P<0.001$ $(95 \% \mathrm{CI}=0.75-0.98)$ (Figure $2 \mathbf{b}$ ).

\section{DISCUSSION}

There are many studies that tried to demonstrate an association between the predictive value of some biomarkers and the risk of BPD in the early stages, but none of them has been effectively used in practice. In the current study, we evaluated the predictive values of IL-33, sST2, and suPAR as regards BPD development in a cohort of preterm babies (with $\leq 32$ wk of gestation) followed in a neonatal intensive care unit. We measured IL-33, sST2, and suPAR concentrations at the time of delivery 


\section{Articles | Tunc et al.}

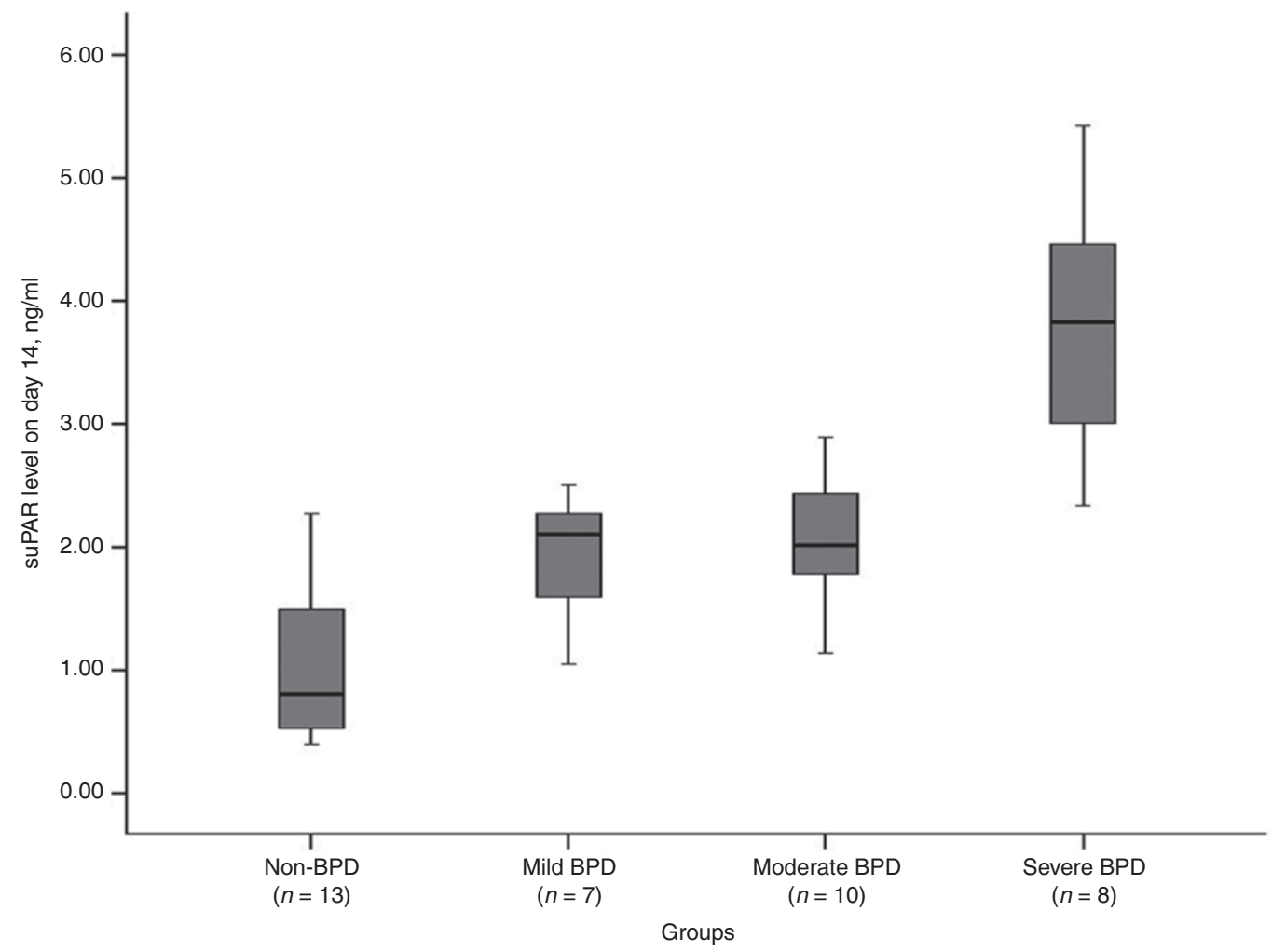

Figure 1. The comparison between PB-suPAR levels on day 14 and the severity of BPD. There was a statistically significant positive correlation between PB-suPAR levels and the severity of BPD $(P<0.001)$. BPD, bronchopulmonary dysplasia; PB, peripheral blood; suPAR, soluble form of the urokinase plasminogen activator receptor.

a

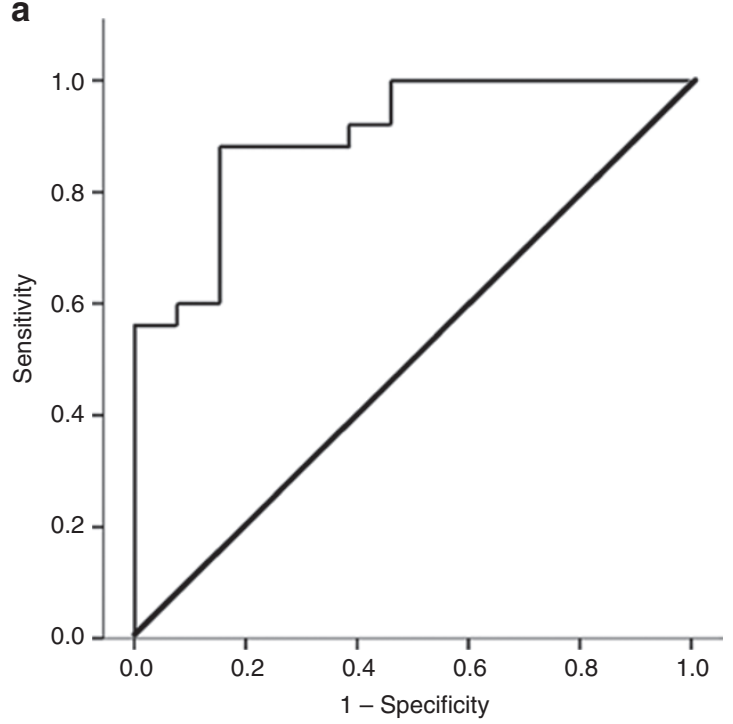

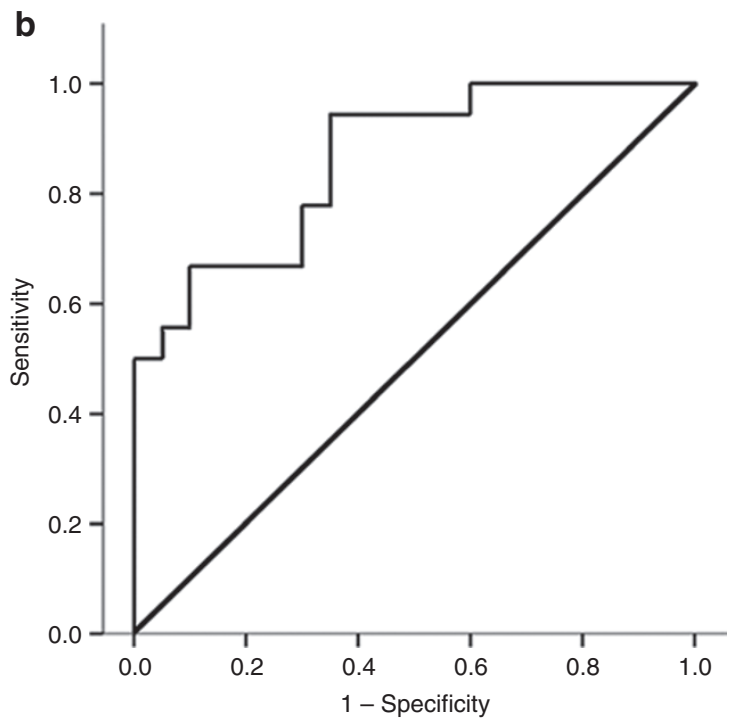

Figure 2. The receiver operating characteristic (ROC) curve analysis for (a) PB-suPAR levels of patients with and without BPD. The area under the curve was $0.902(P<0.001 ; 95 \% \mathrm{Cl}=0.80-0.99)$, (b) PB-suPAR levels comparing two groups (group 1: non-BPD and mild BPD patients vs. group 2: patients with moderate and severe BPD). The area under the curve was $0.861(P<0.001 ; 95 \% \mathrm{Cl}=0.75-0.98)$. BPD, bronchopulmonary dysplasia; $\mathrm{Cl}$, confidence interval; $\mathrm{PB}$, peripheral blood; suPAR, soluble form of the urokinase plasminogen activator receptor.

and on the day 14 of life, in blood from umbilical cord (termed $\mathrm{CB}$ ) and in the peripheral blood (termed $\mathrm{PB}$ ), respectively. There was no correlation between CB-sST2, CB-IL33, and the development of BPD. As compared with the CB-suPAR levels, $\mathrm{PB}$-suPAR levels had a significant value in predicting BPD.
There was a strong positive correlation between PB-suPAR levels and the severity of BPD.

IL-33 and sST2 have recently been found to be involved in the pathogenesis of chronic inflammatory diseases (11). Stolarski et al. demonstrated that the IL-33/ST2 signaling pathway 
activated airway eosinophils, which exacerbate airway inflammation in an autocrine and paracrine manner (19). Yagami et al. showed that the IL-33/ST2 signaling pathway activated the production of IL- 8 by epithelial cells and in this way, could be involved in chronic allergic inflammation of the asthmatic airway (20). In our study, there were no significant differences between BPD and non-BPD groups in terms of CB-IL33 and PB-IL33 levels.

Martinez et al. showed that concentrations of sST2 were significantly higher in patients with pulmonary diseasescompared with the levels in subjects without cardiopulmonary disease-and were high in patients with acute pulmonary diseases, in addition to having prognostic importance for death by $1 \mathrm{y}(21)$. In our study, there was no significant difference between BPD and non-BPD groups in the CB-sST2 levels. However, we found that PB-sST2 levels in the BPD group were statistically higher than the levels in the non-BPD group, and this finding was concordant with literature.

The UPAR and its ligand uPA are participants in various immunologic functions and promote tissue invasion by converting plasminogen into plasmin, resulting in degradation of the extracellular matrix. In some studies, it has been reported that plasma suPAR level is related to different conditions of immune activation and is a reliable prognostic marker for the level of inflammation (22). Additionally, expression and release of $\mathrm{UPAR}$ is upregulated by a number of proangiogenic or proatherogenic growth factors, cytokines such as IL-1, and vascular endothelial growth factor, suggesting its involvement in proliferative and inflammatory processes (23). Koch et al. demonstrated the prognostic impact of serum suPAR levels during the clinical course within the first week in a large cohort of critical adult patients in a medical intensive care unit (24). Sarafidis et al. showed that infants with BPD have an early persistent inflammatory response (25). Some studies have demonstrated that there is an increase in plasma suPAR levels in infectious and rheumatic diseases (23). In our study, the frequencies of PPROM, patent ductus arteriosus, duration of mechanical ventilation, and RDS in the BPD group were significantly higher than the frequencies of the same in the non-BPD group. There was no significant difference between BPD and non-BPD groups for CB-suPAR levels. However, we found that PB-suPAR levels in the BPD group were statistically higher than the levels in the non-BPD group. This increase in PB-suPAR levels may be associated with immunologic and inflammatory mechanisms, playing a role in the pathogenesis of BPD. A recent study showed that plasma suPAR level is positively associated with risk of development of diabetes and cardiovascular disease (17). This finding may mean that high level of suPAR is an indicator of low-grade inflammation similar to BPD. In our study, we found a statistically significant positive correlation between PB-suPAR levels and the severity of BPD. The optimum diagnostic cutoff point was $1.55 \mathrm{ng} / \mathrm{ml}$. The area under the curve was 0.902 , and sensitivity, specificity, positive predictive value, and negative predictive value were 88.0, 84.6, 78.0, and 91.7\%, respectively. We also found that an increase of $1 \mathrm{ng} / \mathrm{ml}$ in PB-suPAR levels increased the risk of BPD 12.92-fold. The risk of BPD development in patients with elevated PB-suPAR levels was 40.33-fold higher than in those with lower levels. There was a statistically significant positive correlation between PB-suPAR levels and the severity of BPD.

van Zoelen et al. showed that UPAR played a detrimental role in hyperoxia-induced lung injury and that uPAR deficiency is associated with diminished neutrophil influx into both lung tissues and bronchoalveolar spaces and is accompanied by decreased pulmonary injury (26). Shushakova et al. showed that the urokinase/urokinase receptor system mediated the immunoglobulin G immune complex-induced inflammation in lungs (27). In the current study, it is unclear whether serum suPAR level is truly causatively involved in mechanisms of $\mathrm{BPD}$ or whether it reflects inflammation in BPD.

In conclusion, in this study, we aimed to evaluate whether IL-33, sST2, and suPAR may be predictors of BPD development. Among these, PB-suPAR levels displayed the best predictive accuracy for development of BPD. Given the high predictive value of the 14 th-d suPAR levels in our results, future studies should address whether suPAR levels can be used to avoid the course of BPD and also for developing preventive strategies. Finally, further studies are definitely required for a better understanding of the biochemical and regulatory roles of suPAR in preterm babies. The limitation of our study is the evaluation of only the predictive value of these markers. To investigate the prognostic significance of plasma suPAR, further studies are needed.

\section{METHODS \\ Subjects}

Preterm infants born in Istanbul University's Faculty of Medicine between 2011 and 2012 and with a gestational age $\leq 32 \mathrm{wk}$ were enrolled in the study. Gestational age was assessed by maternal menstrual dating and obstetric ultrasonography and was confirmed by the new Ballard score (28). Preterm infants of mothers who had diabetes mellitus or parathyroid, bone, renal, gastrointestinal, and other chronic diseases were excluded.

A total of 44 preterm infants were included in the study, but six of them were dead because of several reasons. Demographic findings consisting of gestational age, birth weight, gender, BPD, antenatal betamethasone usage, PPROM, chorioamnionitis, early or late sepsis, maternal antibiotic usage, RDS, duration of mechanical ventilation, patent ductus arteriosus, and necrotizing enterocolitis of groups were recorded from the patients' files. BPD was classified and defined as mild, moderate, and severe according to the National Institute of Child Health and Human Development and National Heart, Lung and Blood Institute Workshop (29). Applying clinical criteria to samples from both $28 \mathrm{~d}$ and $36 \mathrm{wk}$ postmenstrual age, workshop participants defined mild BPD as the need for supplemental oxygen at $\geq 28 \mathrm{~d}$ but not at $36 \mathrm{wk}$ postmenstrual age; moderate BPD was defined as the need for supplemental oxygen at $28 \mathrm{~d}$, in addition to supplemental oxygen at $\mathrm{FiO}_{2}$ (fraction of inspired oxygen) $\leq 0.30$ at $36 \mathrm{wk}$ postmenstrual age; and criteria for severe BPD included the need for supplemental oxygen at $28 \mathrm{~d}$ and, at $36 \mathrm{wk}$ postmenstrual age, the need for mechanical ventilation and/or $\mathrm{FiO}_{2}$ (fraction of inspired oxygen) $>0.30$ (30).

The preterm infants with BPD and the non-BPD infants were compared with each other in terms of demographic and laboratory findings.

\section{Blood Samples}

Blood samples were collected from umbilical cord at the time of delivery for analyses of suPAR (CB-suPAR), sST2 (CB-sST2), and IL-33 (CB-IL33) and from peripheral blood on the day 14 of life (for analyses of PB-suPAR, PB-sST2, and PB-IL33). After collecting 
all samples and clotting of the same, the serum samples were frozen and analyzed. Plasma levels of IL-33 (GenWay Biotech, San Diego, CA) were measured by commercial sandwich enzyme-linked immunosorbent assay. Serum levels of sST2 (The Marine Biological Laboratory, Woods Hole, MA) were measured by commercial sandwich enzyme-linked immunosorbent assay. The capture antibody and the horseradish peroxidase-conjugated detection antibody involve two anti-human ST2 mAbs (monoclonal antibodies) that recognize two different epitopes. The sensitivity of the immunoassays for IL-33 and sST2 are 0.7 and $0.032 \mathrm{ng} / \mathrm{ml}$, respectively. The levels of suPAR were measured by an enzyme-linked immunosorbent assay (detection limit: $0.25 \mathrm{ng} / \mathrm{ml}$ ), as specified by the manufacturer (American Diagnostica, Greenwich, CT).

\section{Statistical Analysis}

SPSS for Windows was used for data analysis on a personal computer. Mann-Whitney $U$-test was used for comparing continuous variables, and $\chi^{2}$ test was used for nominal variables. Kruskal-Wallis and posthoc Bonferroni-adjusted Mann-Whitney $U$-test were used for comparing intergroup variances through BPD as mild-moderate and severe. We calculated odds ratio values by logistic regression analysis for suPAR after adjusting for confounders, such as PPROM, RDS, patent ductus arteriosus, duration of mechanical ventilation, gender, and chorioamnionitis. Spearman correlation test was used to determine the linear association between BPD severity and suPAR levels. A $P$ value $<0.05$ was considered statistically significant.

Istanbul University School of Medicine Ethics Committee approved the study protocol, and written informed consents was obtained from the parents before the blood samples were taken.

\section{ACKNOWLEDGMENTS}

We thank our colleagues and FMF Arthritis Vasculitis and Orphan disease Research in Paediatric Rheumatology (FAVOR) for their helpful contributions and suggestions.

\section{STATEMENT OF FINANCIAL SUPPORT}

No financial assistance was received in support of the study.

Disclosure: The authors do not have any conflict of interest to disclose.

\section{REFERENCES}

1. Kinsella JP, Greenough A, Abman SH. Bronchopulmonary dysplasia. Lancet 2006;367:1421-31.

2. Ambalavanan N, Carlo WA. Ventilatory strategies in the prevention and management of bronchopulmonary dysplasia. Semin Perinatol 2006;30:192-9.

3. Kul M, Saldır M, Gulgun M, Kesik V, Sarıcı SU, Alpay F. Retrospective evaluation of low birth weight newborns followed up with the diagnosis of respiratory distress syndrome in the newborn intensive care unit. Gulhane Med J 2005;47:290-3.

4. Bhandari V, Finer NN, Ehrenkranz RA, et al; Eunice Kennedy Shriver National Institute of Child Health and Human Development Neonatal Research Network. Synchronized nasal intermittent positive-pressure ventilation and neonatal outcomes. Pediatrics 2009;124:517-26.

5. Ehrenkranz RA, Das A, Wrage LA, et al; Eunice Kennedy Shriver National Institute of Child Health and Human Development Neonatal Research Network. Early nutrition mediates the influence of severity of illness on extremely LBW infants. Pediatr Res 2011;69:522-9.

6. Sarici SU, Kul M, Candemir G, Gursel O, Alpay F, Gokcay E. Inhaled nitric oxide in a preterm newborn with severe hypoxemic respiratory failure. Gulhane Med J 2004;46:255-7.

7. Reyburn B, Martin RJ, Prakash YS, MacFarlane PM. Mechanisms of injury to the preterm lung and airway: implications for long-term pulmonary outcome. Neonatology 2012;101:345-52.

8. Aghai ZH, Saslow JG, Mody K, et al. IFN- $\gamma$ and IP-10 in tracheal aspirates from premature infants: relationship with bronchopulmonary dysplasia. Pediatr Pulmonol 2013;48:8-13.

9. Wright CJ, Kirpalani H. Targeting inflammation to prevent bronchopulmonary dysplasia: can new insights be translated into therapies? Pediatrics 2011;128:111-26.
10. Oshikawa K, Yanagisawa K, Tominaga Si, Sugiyama Y. ST2 protein induced by inflammatory stimuli can modulate acute lung inflammation. Biochem Biophys Res Commun 2002;299:18-24.

11. Xu D, Jiang HR, Kewin P, et al. IL-33 exacerbates antigen-induced arthritis by activating mast cells. Proc Natl Acad Sci U S A 2008;105:10913-8.

12. Sanada S, Hakuno D, Higgins LJ, Schreiter ER, McKenzie AN, Lee RT. IL33 and ST2 comprise a critical biomechanically induced and cardioprotective signaling system. J Clin Invest 2007;117:1538-49.

13. Alves-Filho JC, Sônego F, Souto FO, et al. Interleukin-33 attenuates sepsis by enhancing neutrophil influx to the site of infection. Nat Med 2010;16:708-12.

14. May AE, Schmidt R, Kanse SM, et al. Urokinase receptor surface expression regulates monocyte adhesion in acute myocardial infarction. Blood 2002;100:3611-7.

15. Fazioli F, Resnati M, Sidenius N, Higashimoto Y, Appella E, Blasi F. A urokinase-sensitive region of the human urokinase receptor is responsible for its chemotactic activity. EMBO J 1997;16:7279-86.

16. Fernebro E, Madsen RR, Fernö M, et al. Prognostic importance of the soluble plasminogen activator receptor, suPAR, in plasma from rectal cancer patients. Eur J Cancer 2001;37:486-91.

17. Eugen-Olsen J, Andersen O, Linneberg A, et al. Circulating soluble urokinase plasminogen activator receptor predicts cancer, cardiovascular disease, diabetes and mortality in the general population. J Intern Med 2010;268:296-308.

18. Sehestedt T, Lyngbæk S, Eugen-Olsen J, et al. Soluble urokinase plasminogen activator receptor is associated with subclinical organ damage and cardiovascular events. Atherosclerosis 2011;216:237-43.

19. Schrama AJ, Bernard A, Poorthuis BJ, Zwinderman AH, Berger HM, Walther FJ. Cord blood Clara cell protein CC16 predicts the development of bronchopulmonary dysplasia. Eur J Pediatr 2008;167:1305-12.

20. Stolarski B, Kurowska-Stolarska M, Kewin P, Xu D, Liew FY. IL-33 exacerbates eosinophil-mediated airway inflammation. J Immunol 2010;185:3472-80.

21. Martinez-Rumayor A, Camargo CA, Green SM, Baggish AL, O’Donoghue M, Januzzi JL. Soluble ST2 plasma concentrations predict 1-year mortality in acutely dyspneic emergency department patients with pulmonary disease. Am J Clin Pathol 2008;130:578-84.

22. Eugen-Olsen J, Gustafson P, Sidenius N, et al. The serum level of soluble urokinase receptor is elevated in tuberculosis patients and predicts mortality during treatment: a community study from Guinea-Bissau. Int J Tuberc Lung Dis 2002;6:686-92.

23. Kofoed K, Eugen-Olsen J, Petersen J, Larsen K, Andersen O. Predicting mortality in patients with systemic inflammatory response syndrome: an evaluation of two prognostic models, two soluble receptors, and a macrophage migration inhibitory factor. Eur J Clin Microbiol Infect Dis 2008;27:375-83.

24. Koch A, Voigt S, Kruschinski C, et al. Circulating soluble urokinase plasminogen activator receptor is stably elevated during the first week of treatment in the intensive care unit and predicts mortality in critically ill patients. Crit Care 2011;15:R63.

25. Sarafidis K, Drossou-Agakidou V, Kanakoudi-Tsakalidou F, et al. Evidence of early systemic activation and transendothelial migration of neutrophils in neonates with severe respiratory distress syndrome. Pediatr Pulmonol 2001;31:214-9.

26. van Zoelen MA, Florquin S, de Beer R, et al. Urokinase plasminogen activator receptor-deficient mice demonstrate reduced hyperoxia-induced lung injury. Am J Pathol 2009;174:2182-9.

27. Shushakova N, Eden G, Dangers M, et al. The urokinase/urokinase receptor system mediates the IgG immune complex-induced inflammation in lung. J Immunol 2005;175:4060-8.

28. Vik T, Vatten L, Markestad T, Jacobsen G, Bakketeig LS. Dubowitz assessment of gestational age and agreement with prenatal methods. Am J Perinatol 1997;14:369-73.

29. Ehrenkranz RA, Walsh MC, Vohr BR, et al; National Institutes of Child Health and Human Development Neonatal Research Network. Validation of the National Institutes of Health consensus definition of bronchopulmonary dysplasia. Pediatrics 2005;116:1353-60.

30. Jobe AH, Bancalari E. Bronchopulmonary dysplasia. Am J Respir Crit Care Med 2001;163:1723-9. 